\title{
e-Migrinter
}

16 | 2017

Récits d'exilés

\section{" Au milieu du chemin de notre vie »}

Le témoignage dans un centre de soins pour exilés à Paris : contenu, formes et intrigue

\section{Giacomo Mantovan}

\section{(2) OpenEdition}

Journals

Édition électronique

URL : https://journals.openedition.org/e-migrinter/948

DOI : $10.4000 /$ e-migrinter.948

ISSN : 1961-9685

Éditeur

UMR 7301 - Migrinter

Référence électronique

Giacomo Mantovan, « « Au milieu du chemin de notre vie » , e-Migrinter [En ligne], 16 | 2017, mis en ligne le, consulté le 20 mai 2021. URL : http://journals.openedition.org/e-migrinter/948 ; DOI : https:// doi.org/10.4000/e-migrinter.948

Ce document a été généré automatiquement le 20 mai 2021.

Tous droits réservés 


\section{«Au milieu du chemin de notre vie »}

Le témoignage dans un centre de soins pour exilés à Paris : contenu, formes et intrigue

\section{Giacomo Mantovan}

Cette enquête a été financée par l'EHESS, et la rédaction de l'article par l'International Institute for Asian Studies (Leyde). Je remercie Frédérik Detue, Raphaëlle Guidée et Anouche Kunth de m'avoir invité à intervenir lors de la journée d'étude "Récits d'exilés ", à l'Université de Poitiers, les 30 et 31 mars 2016, participation qui est à la base de cet article. Ma gratitude va également au directeur général Arnaud Veïsse et toute l'équipe du COMEDE pour m'avoir permis d'y mener ma recherche. Enfin, je remercie tous les usagers du COMEDE qui ont accepté de participer à cette étude.

\footnotetext{
Nel mezzo del cammin di nostra vita mi ritrovai per una selva oscura, ché la dritta via era smarrita. Ahi quanto a dir qual era è cosa dura Esta selva selvaggia e aspra e forte Che nel pensier rinova la paura! Dante Alighieri, Inferno, Canto 1, 1-6. ${ }^{1}$
}

\section{Introduction}

Pour les demandeurs d'asile présents sur le sol français, le passage par un centre de soins pour migrants est devenu une étape courante du parcours. Suivant des approches cliniques différentes (Fassin et d'Halluin, 2007), ces centres offrent des soins médicaux et psychologiques gratuits aux exilés, en particulier à ceux qui ont été victimes de violence politique. Toutefois, si nombre de requérants s'adressent à ces centres notamment au Comité médical pour les exilés (COMEDE) pris pour enquête dans cet article - c'est non seulement pour y recevoir des soins, mais également pour obtenir un 
certificat attestant qu'ils ont bien été victimes de violence et de torture. Or ce document s'avère indispensable pour constituer le dossier de la demande d'asile.

2 Si les témoignages ont toujours été confrontés à la question de la vérité (Detue et Lacoste, 2016), celle-ci devient un enjeu crucial dans le cas des demandeurs d'asile : la crédibilité de leur histoire est l'élément primordial pour la réussite de leur demande. Lors des étapes de leur parcours administratif, les exilés sont amenés à exposer leur histoire à plusieurs reprises devant différentes audiences (Office de protection des réfugiés et apatrides (OFPRA), Cour nationale du droit d'asile (CNDA), «écrivains publics ", médecins) et sous diverses formes (récits écrits, réponses orales aux questions des examinateurs, documents, certificats médicaux). Dans ce contexte, la demande de certificat médical au COMEDE représente une étape supplémentaire du parcours, au cours duquel est façonnée une image des requérants destinée à être exposée en public. Les récits produits deviennent ainsi le matériel argumentatif permettant aux institutions françaises de répondre aux questions que pose la présence des étrangers à la société d'accueil: qui sont tous ces demandeurs d'asile? Ont-ils vraiment besoin d'être protégés par la France, ou bien viennent-ils ici pour profiter de son welfare state?

Les deux études de cas présentées ici - la production des certificats médicaux et le récit d'un patient qui, lors d'une visite à mon domicile, m'a raconté en détail son parcours nous aideront à appréhender la manière dont la "politique de la preuve " (Fassin et Rechtman, 2007) façonne la forme et le contenu des récits, et influe fortement sur le rôle du COMEDE. Centrant l'attention sur le contexte qui requiert le témoignage plutôt que sur le contenu de celui-ci, la seconde étude de cas montrera que les injonctions sociales de la demande d'asile modifient une règle de la théorie narrative, selon laquelle les narrations s'orientent toujours vers une conclusion.

\section{Méthodologie}

4 Cet article se base sur une enquête menée au COMEDE de mars à juillet 2009. Celle-ci a été pensée comme une partie d'un projet visant à saisir la manière dont les institutions d'accueil façonnent les récits des demandeurs d'asile d'origine tamoule sri-lankaise. Bien que centrée sur la population tamoule, cette recherche aborde des questions et des situations auxquelles sont confrontées les demandeurs d'asile de toute origine. La direction du COMEDE m'a autorisé à assister aux consultations des médecins généralistes et à m'entretenir avec des patients dont j'avais préalablement sollicité l'accord, que j'ai pu rencontrer hors du COMEDE (souvent chez moi) pour discuter de leur parcours en détail et dans le calme. Les données comprennent l'observation de plusieurs dizaines de consultations médicales; la réalisation de dix entretiens avec des patients et trois avec des médecins; enfin, l'analyse des documents et des « rapports d'activités » rédigés par le COMEDE.

\section{Le COMEDE, entre soins et défense des droits des exilés}

5 Le COMEDE est une association fondée en 1979 ayant pour but de fournir des soins aux exilés et de défendre leurs droits. Il s'adresse principalement aux étrangers en situation 
irrégulière, ou dotés d'un statut juridique précaire, qui rencontrent des difficultés dans l'accès aux soins. Il propose des consultations médicales et psychologiques gratuites et réalise, en partenariat avec la Cimade, un accompagnement social et juridique. Il mène également des travaux de recherche, de formation et d'information, et publie la revue trimestrielle Maux d'exil.

Un bref rappel historique concernant les exilés tamouls dont il est question ici : ils ont fui la guerre civile qui a opposé, entre 1983 et 2009, le gouvernement sri lankais aux Liberation Tigers of Tamil Eelam (LTTE), un mouvement issu de la minorité tamoule luttant pour l'indépendance. Cette guerre, où la violation répétée des droits de l'homme (torture, enlèvements, viols, assassinats, etc.) est amplement documentée, s'est conclue en mai 2009 par le massacre de dizaines de milliers de civils, l'ONU allant jusqu'à envisager l'hypothèse de 40000 morts (ONU, 2011). Pendant plusieurs années, les Sri Lankais - dont presque tous sont Tamouls - représentent la nationalité qui recourt le plus aux services du COMEDE : plus de 4000 entre 2003 et 2010 (COMEDE, 2011, p. 16). Dans cette population on relève des pourcentages très élevés d'antécédents de violences et de tortures: entre 2007 et 2010, les médecins constatent un taux d'antécédents de « violences » de $75 \%$ et de «tortures » de $51 \%$ (COMEDE, 2011, p. 22). Il s'agit d'une population souffrant d'une "vulnérabilité structurelle ", c'est-à-dire une condition de violence et de détresse qui, pouvant aller de l'exploitation économique et politique au racisme et au sexisme, a un impact négatif sur la subjectivité des individus (Quesada, Hart, Bourgois, 2001). En fait, par rapport à leurs compatriotes arrivés dans les années 1980 et 1990, les Tamouls sri lankais qui émigrent depuis les années 2010 sont plus démunis : issus principalement des castes et classes moins aisées, la plupart sont dans une situation financière extrêmement difficile, et leur condition de santé est souvent mauvaise en raison des blessures, physiques et mentales, qui résultent de l'expérience de la guerre civile et de la situation actuelle d'extrême précarité.

\section{Un lieu de témoignage}

7 Cet extrait de mon cahier de terrain contribuera à illustrer les consultations avec les médecins.

Le patient est un homme d'environ 30 ans, qui a déjà été examiné par ce même médecin il y a trois semaines. Il souffre de dépression. Il explique être très triste suite au décès de sa mère (complications rénales) et à la disparition de son frère au Sri Lanka (ces deux événements sont récents). Il raconte qu'il a été arrêté et torturé. Il dit avoir envie de mourir, de se suicider, cependant il n'a jamais pensé aux moyens de passer à l'acte.

La fois précédente le médecin avait prescrit des antidépresseurs ; il l'interroge sur les effets des médicaments ; le patient répond que le traitement fonctionne un peu, mais qu'il se sent toujours aussi triste, qu'il dort beaucoup, que quand il est éveillé il est très angoissé, il pense constamment au Sri Lanka, il est inquiet pour son frère dont il n'a toujours pas de nouvelles. Il assiste au cours de français organisé par le CADA [Centre d'accueil de demandeurs d'asile] où il est hébergé, il s'y rend seulement les jours où il n'est pas trop déprimé, mais il se demande à quoi ça sert d'étudier le français s'il n'a pas de papiers... 
Aujourd'hui il est venu voir le médecin parce qu'il se sent mal, mais quand il sera convoqué par la CNDA, il sollicitera le certificat médical ; il a déjà un certificat établi par un autre médecin.

Le médecin lui propose une consultation chez le psychologue du COMEDE, le patient accepte.

Ensuite, il demande au médecin d'examiner ses jambes, où il a mal : sur les chevilles il y a des traces sombres, il a moins mal quand il marche lentement ou qu'il est assis ; un autre médecin lui avait donné des médicaments pour cela. Il se plaint également de douleurs aux oreilles, où il a été frappé violemment par des militaires, il a beaucoup saigné ; il a déjà exposé ce problème à un autre généraliste, qui lui a recommandé de consulter un spécialiste ORL, mais il n'a pas encore demandé un rendez-vous. Le médecin l'examine et lui prescrit des médicaments.

(Extrait de cahier de terrain, 30 mars 2009).

Dans ces notes on relève d'emblée trois éléments récurrents dans les consultations effectuées au COMEDE : en premier lieu, les troubles du patient étant dus à la violence de la guerre et aux problèmes sociaux et légaux relatifs à l'installation en France, l'exilé raconte brièvement son histoire et ses problèmes, notamment les persécutions subies: dès lors, la relation avec le médecin devient un acte de témoignage; en deuxième lieu, les souffrances psychologiques causées par la torture poursuivent la victime plusieurs années après les faits; enfin, le patient a besoin d'un certificat médical attestant les tortures et les violences subies.

\section{... et de certification des violences subies}

La demande du certificat est très commune du fait que les institutions d'accueil recommandent aux requérants qui déclarent avoir subi des violences ou des tortures d'étayer leurs dires par l'autorité d'un spécialiste. Depuis longtemps des certificats médicaux ont été présentés à l'OFPRA et à la Commission de recours des réfugiés (CRR) ${ }^{2}$ (Noiriel, 1991), mais à partir de la fin des années 1980 on observe une augmentation considérable de leur nombre ; aujourd'hui le certificat médical est devenu une pièce habituellement incluse dans le dossier de demande d'asile. Bien que tout médecin généraliste soit habilité à effectuer une expertise médicale, la pratique courante des requérants est de recourir pour cela aux centres médicaux pour exilés; ceux-ci sont d'ailleurs considérés comme plus crédibles par nombre d'examinateurs de l'OFPRA et de la CNDA3 .

L'expertise médicale est réalisée en l'espace de deux ou trois consultations d'environ 30-35 minutes. Lors de la première, le médecin fait connaissance avec le patient, s'informe de ses problèmes de santé et de ses difficultés liées à l'installation en France (recherche de logement, problèmes bureaucratiques pour l'accès aux soins, etc.) et termine en suggérant au patient de faire un «bilan de santé ». L'établissement du certificat médical n'advient jamais lors de la première consultation, mais lors de la deuxième ou la troisième. 
11 Durant l'expertise les patients relatent de façon succincte leur histoire personnelle, exposent les traces de leurs blessures, expliquent comment celles-ci ont été causées, et précisent s'ils ressentent toujours des douleurs. Le médecin questionne le patient sur les éléments nécessaires pour mettre en récit les signes constatés: où, quand et comment les faits ont-ils eu lieu ? Le modèle de certificat décrit par le Guide pratique 2008 du COMEDE comprend deux parties (COMEDE, 2008, p. 381). La première porte sur les déclarations du patient : ici, il s'agit de reprendre les motifs des persécutions, les éléments, les doléances et les constatations de l'examen (ce qui peut concerner les circonstances et les conditions des sévices et de la détention). La seconde partie est réservée à la constatation de l'examen clinique et/ou à la prise en charge du patient : conclusion de l'interrogatoire, les doléances du patient (plaintes somatiques et psychologiques), éventuelle présence du Posttraumatic Stress Desorder (PTSD), conclusions de l'examen physique: localisation et caractéristiques des cicatrices et autres séquelles traumatiques. Dans la conclusion, le médecin atteste la probable véracité du récit du requérant: "Ces constatations sont compatibles avec les déclarations de l'intéressé(e)»(COMEDE, 2008, p. 381). Les déclarations du patient sont à formuler au conditionnel, les signes corporels ou psychiques ne pouvant pas être considérés comme une preuve certaine de la véracité du récit.

12 Au sujet de l'expertise médicale ${ }^{4}$ on peut formuler les considérations suivantes. En premier lieu, la production de certificats est le symptôme de la crise du droit d'asile, laquelle se manifeste, d'abord, par la méfiance envers la parole des requérants. Produisant un certificat médical, le médecin se substitue à la parole du patient. Conscients de ce problème, les médecins expriment leur malaise :

\begin{abstract}
Je me substitue à leur parole, on leur vole leur parole. Ce qu'écrit le médecin c'est vrai, ce qu'ils [les requérants] racontent ... [il fait un geste de désespoir] c'est soumis à caution, on va dire... voilà. C'est terrible le certificat comme ça! (Entretien avec un médecin, Le Kremlin-Bicêtre, le 05/06/2009).
\end{abstract}

Dans l'expertise médicale, le médecin n'est plus chargé de soigner le patient, mais d'attester une vérité qui, faute de confiance en la parole des requérants, réside désormais dans leur corps ou dans leur psyché (Fassin et d'Halluin, 2005).

Une conséquence de cette méfiance est que les demandeurs d'asile en arrivent à penser qu'en l'absence de " preuves » leur récit ne sera pas cru par les agents de l'OFPRA et la CNDA. Lors de mon travail de terrain, à plusieurs reprises j'ai entendu des requérants affirmer qu'ils pouvaient prouver, avec des certificats ou des documents, certains épisodes de leur histoire. Celui qui a moins de " preuves » peut être plus pessimiste sur la réponse à sa demande, comme le commentait un de mes interlocuteurs :

Pour prouver une histoire à l'OFPRA il faut [avoir] des preuves [...]. La seule preuve que j'ai c'est une cicatrice sur le crâne, quand l'armée m'a frappé (entretien avec un demandeur d'asile, Paris, le 30/04/2009).

14 En deuxième lieu, la quête du certificat peut entraver le processus thérapeutique. En effet, les contraintes administratives, ayant des temporalités et des exigences différentes de celles du soin, peuvent réactiver la souffrance psychologique, faire revivre les sévices infligés par les bourreaux et compliquer la prise en charge thérapeutique (COMEDE, 2008, p. 240, p. 248). Aux yeux des médecins, l'expertise peut se révéler violente et contraire aux temporalités et aux modalités qui seraient nécessaires pour une prise en charge thérapeutique efficace : 
Le problème - explique un médecin - c'est que le temps du certificat n'est pas le temps du soin: le temps du soin est beaucoup plus long et beaucoup plus prudent. L'entretien n'est pas le même (entretien avec un médecin, Le Kremlin-Bicêtre, le 03/06/2009).

En dernier lieu, l'expertise "vide » les expériences et les histoires personnelles et collectives. Le fait de devoir révéler l'indicible en l'espace de deux ou trois consultations médicales constitue un processus violent, et les patients cherchent donc à se protéger :

Il faut aussi - commente un médecin - se mettre à l'abri, il faut se protéger quand on a été torturé. C'est très difficile [car] il y a une effraction quand quelqu'un est torturé, il y a effraction dans la personnalité, dans le physique et le psychique, et les gens se protègent en en parlant le moins possible. Ils se protègent parce que [la torture] c'est indicible (entretien avec un médecin, Le Kremlin-Bicêtre, le 03/06/2009).

C'est en ce sens que j'interprète l'apparent manque d'émotions observé chez les requérants durant l'exposé de leurs blessures. Durant la narration ils se distancient comme s'il n'y avait pas une identification complète entre la personne qui raconte et les faits évoqués. Commentant cette manière de relater les épreuves subies, un médecin va jusqu'à évoquer le fait que leurs récits pourraient sembler "stéréotypés » :

Ils prennent de la distance dans la narration. Des fois, ils racontent ça comme si c'était arrivé à quelqu'un d'autre. Quand on me dit: "Là, j'ai été en prison, on m'a fait ceci, on m'a fait cela", j'ai la sensation que les gens qui s'expriment ainsi, ce sont ceux qui cherchent un certificat médical. La demande de soin est très différente (entretien avec un médecin, Le Kremlin-Bicêtre, le 05/06/2009).

En fait, pour réussir à dire les sévices subis, l'exilé essaye de s'éloigner de son être intime, il raconte son vécu presque comme s'il parlait de quelqu'un d'autre.

\section{Devenir un témoin}

Selon un médecin, la rumeur circule au sein de la communauté tamoule que pour recevoir l'asile il faut se rendre au COMEDE et y obtenir un certificat. Plusieurs médecins m'ont dit regretter le fait que nombre d'exilés tamouls, une fois effectuée l'expertise médicale et obtenu le certificat requis, ne reviennent plus au Comité, alors que ces patients auraient eu besoin de se faire traiter (entretien avec trois médecins, Le Kremlin-Bicêtre, plusieurs dates). Lors des consultations auxquelles j'ai assisté deux comportements ont attiré mon attention: d'une part, certains patients se lançaient dans le récit des violences et tortures subies dès le début de la consultation, avant même de préparer le certificat; d'autre part, alors que je cherchais à établir la conversation avec des patients dans la salle d'attente, certains mentionnaient les violences subies sans que j'aie posé la moindre question à ce sujet - or un tel comportement n'avait pas lieu lorsque je les rencontrais dans d'autres contextes. L'angoisse due à la réduction des taux d'octroi de l'asile, la mauvaise connaissance des procédures de la demande d'asile et de la différence de statut entre l'OFPRA, la CNDA, le COMEDE et un chercheur universitaire peut mener nombre de requérants à se poser, dans tous ces contextes, comme une victime à la recherche d'une reconnaissance. 
17 D'autre part, les Tamouls, entre eux, parlent très peu des injustices subies. Les nombreuses épreuves traversées sont souvent considérées comme quelque chose dont tous ont fait l'expérience, et pour cette raison ils ne se les racontent pas les uns aux autres. De surcroit, la mémoire des violences de la guerre est entièrement laissée aux associations nationalistes qui, en se l'appropriant, l'insèrent dans des revendications politiques, tandis qu'elle est tue dans les familles: probablement pour protéger les enfants, les parents ne leur racontent pas leur histoire. Par ailleurs, la période de la demande d'asile n'est pas seulement marquée par les problèmes précédemment décrits, elle est aussi porteuse d'espoir, en particulier pour les jeunes qui attendent de pouvoir profiter de la qualité de vie qu'offre la France, une fois les papiers obtenus. Toutefois, dans les situations concrètes que j'ai observées, force est de constater que de tels espoirs et attentes positives sont passés sous silences: car dans un contexte où le témoin cherche une reconnaissance institutionnelle, ces expectatives personnelles ne trouvent pas à se dire.

Ce ne sont pas les persécutions qui font le témoin, mais le contexte qui requiert sa mise en récit ; de fait, le désir spontané de témoigner s'avère assez rare chez les Tamouls. Le témoignage est donc ici le fruit d'une injonction sociale (Artières, Farge, Laborie, 2002) façonnée par les rapports de pouvoir entre témoin et audience. À chaque nouvelle rencontre au COMEDE je veillais d'emblée à préciser au requérant que, vu ma qualité de chercheur universitaire, ce qu'il allait me raconter ne pourrait avoir aucun impact sur sa demande d'asile: malgré cette précaution, j'étais quand même perçu comme quelqu'un qui s'intéressait aux souffrances et aux injustices endurées par les requérants. C'est sans doute pour cette raison que le message véhiculé par leur témoignage était identique, même s'il pouvait être parfois plus riche en détails, à celui délivré lors des consultations au COMEDE. On ne doit donc pas s'étonner si nombre de témoignages recueillis dans des relations similaires entre interlocuteur et audience présentent les mêmes caractéristiques : effacement - ou minimisation - des activités politiques (susceptibles d'être mal vues), caractéristiques culturelles et historiques assez réduites, toute l'attention étant portée sur la violence subie, la souffrance passée et présente, et la demande de réhabilitation juridique, médicale et sociale.

\section{Épreuves de vie et mise en récit pour la preuve}

Au moment de notre rencontre au COMEDE, Selva est très angoissé : arrivé en France en janvier 2009, il attend depuis quatre mois la convocation à l'OFPRA, et sa famille lui manque beaucoup. Par la suite, il vient chez moi en apportant son dossier de demande d'asile : il n'a pas très bien saisi mon rôle, lequel lui devient plus clair au fur et à mesure de notre entretien.

Deuxième des trois fils d'une famille originaire de la région de Jaffna, il perd son père à l'âge de dix ans. Sa famille se trouve alors dans une situation matérielle difficile et Selva interrompt ses études très tôt pour aller travailler. Il est embauché dans une fabrique de munitions des LTTE. En 1994, à l'âge de dix-neuf ans, il se marie; l'année suivante naît sa première fille. Trois mois plus tard, sa mère décède. Ayant un bébé de trois mois, Selva et son épouse décident de ne pas se réfugier dans la région du Vanni, comme le conseillent les LTTE à toute la population tamoule lors de la reconquête de Jaffna par l'armée sri lankaise. Selon le récit de Selva, une fois entrée dans la ville, 
l'armée tire sur la population civile, les LTTE s'étant déjà retirés vers le Vanni. Quand la famille peut enfin quitter son abri, le spectacle dans les rues est horrible :

C'est en marchant sur des cadavres qu'on avançait dans la rue. Tous les gens qu'ils voyaient, ils leur ont tiré dessus, tous ont été tués (entretien avec Selva, Paris, le 26/05/2009).

En septembre 1996, lors d'un contrôle de l'armée, Selva est arrêté, puis torturé. Un de ses amis disparaît alors qu'il tente de se rendre à Colombo.

21 La fabrique de munitions des LTTE n'existant plus, Selva apprend le métier de photographe. Il réussit bien dans sa nouvelle profession et acquiert une certaine aisance matérielle. Malgré les difficultés de tous ordres liées au conflit, son épouse et lui mènent leur vie sans penser à émigrer. Au cours de cette période, ils ont deux autres enfants. Malgré la prise de contrôle de la totalité de la péninsule de Jaffna par l'armée sri lankaise, les LTTE réussissent à rétablir des liens avec la population de Jaffna. À partir du cessez-le-feu signé en février 2002, ayant obtenu le droit d'ouvrir des bureaux politiques et d'organiser des événements culturels, ils renforcent leur présence dans la péninsule. Selva, qui est en contact avec cette organisation, réalise divers travaux photographiques pour elle.

En 2006, le conflit se réactive. Selva est arrêté et torturé pendant trois jours en raison de ses activités. Dès sa libération, il porte plainte au bureau des Droits de l'homme et à la Croix-Rouge, car ainsi : on a une preuve qu'on a eu des problèmes et qu'on craint pour sa vie (entretien avec Selva, Paris, le 26/05/2009). En 2007, un événement va précipiter les choses et susciter en lui l'idée de quitter le pays. Un de ses amis, accusé de collaboration avec l'armée sri lankaise, va être éliminé par les LTTE. Selva l'apprend et tente de les en dissuader. Cet épisode, que Selva me relate avec une grande précision, se conclut tragiquement : l'ami est exécuté par des "inconnus " ${ }^{5}$ et Selva est arrêté par l'armée, qui a pris connaissance de son implication dans cette affaire.

\footnotetext{
Un mois après - raconte-il - l'armée est venue me chercher. [...] J'ai été enfermé dans un camp. J'ai des traces sur le corps prouvant que j'ai été torturé. [...] Dans le camp ils m'ont accusé: "Tu as travaillé de telle année à telle année avec les Tigres" (entretien avec Selva, Paris, le 26/05/2009).
}

Après quelque temps il est libéré, mais un jour, alors qu'il se rend au studio en moto avec un ami qui vient d'accomplir une période de détention carcérale à Colombo, deux inconnus ouvrent le feu sur eux, l'ami est tué. Terrorisé, Selva part se cacher chez son frère ; il y restera trois mois. En son absence, quelqu'un se rend chez lui à sa recherche, sans s'identifier, l'épouse de Selva ignore de qui il s'agit... Après ces événements, il part à l'étranger.

24 La narration portant sur le déroulement de l'histoire au Sri Lanka terminée, le récit change complètement, comme s'il était divisé en deux parties. Lorsqu'on aborde le sujet de sa vie en France, Selva semble perdre tout contrôle de soi, son visage et sa parole sont empreints de tristesse, il est au bord des larmes. Depuis qu'il est ici, il est en grande souffrance, il n'arrête pas de penser à sa vie passée, à son pays, à sa famille :

Depuis que je suis arrivé, je ne cesse pas de réfléchir. J'ai toujours voulu retourner; avec ce genre de pensée je n'arrive pas à rester ici, j'ai envie de retourner. S'il n'y avait pas ces problèmes dans ma vie, je retournerais. [...] Je ne dors pas, je ne m'endors pas avant deux ou trois heures du matin. Et à six heures je suis déjà debout. [...] J'essaie d'oublier, mais je n'y arrive pas. Chaque fois que je ferme les yeux pour 
m'endormir, à chaque fois ce sont ces événements-là qui me reviennent, je n'arrive pas à oublier. [...] De temps en temps j'ai des tremblements, et c'est une espèce de terreur qui me prend soudainement (entretien avec Selva, Paris, le 26/05/2009).

J'ai un sentiment de colère, de dégoût. [...] Des fois, quand je pense à ma vie, la seule chose qui fait passer ça, c'est de pleurer. [...] C'est injuste pour un homme d'être séparé de sa famille. Pourquoi c'est comme ça? Je ne comprends pas. Pourquoi est-ce que cette situation arrive? (entretien avec Selva, Paris, le 26/05/2009).

L'attente de la convocation de l'OFPRA et l'impossibilité qui en résulte de savoir ce qu'il adviendra de lui et de sa famille ne permettent pas à Selva d'imaginer un futur meilleur; l'avenir ne pouvant s'envisager, c'est alors le passé qui revient continuellement sous forme de souvenirs, de doutes, d'incertitude. Il ne contrôle pas son destin et n'arrive pas à trouver un sens à sa situation. En évoquant sa famille, Selva ne réussit plus à contrôler son émotion, il éclate en sanglots. Je propose d'arrêter l'entretien, il répond que non, qu'il veut continuer. Il respire profondément et poursuit son récit :

\begin{abstract}
Comme pour tous les pères et mères rencontrés au cours de mon travail de terrain au COMEDE, le plus douloureux et angoissant pour Selva est la séparation d'avec sa famille, elle est constamment au centre de ses pensées :
\end{abstract}

\begin{abstract}
Moi, je sais ce que c'est de ne pas avoir ses parents depuis l'enfance ; j'ai perdu mes parents très jeune, et je sais ce que mes enfants sont en train de vivre aujourd'hui. Aujourd'hui ce sont mes enfants qui me manquent. [...] J'ai trois enfants, deux filles et un garçon. Le dernier a quatre ans. Je parlais, je jouais tout le temps avec lui, et je n'arrive pas à l'oublier. Quand je leur téléphone, il me demande de venir, "Viens, viens! ", me dit-il, mais comment est-ce que je peux y aller ? (entretien avec Selva, Paris, le 26/05/2009).
\end{abstract}

\title{
Contenu et formes des témoignages, la disparition de l'intrigue
}

À observer de plus près la narration de Selva, on y discerne une particularité commune aux récits de nombreux requérants arrivés en France récemment, c'est-à-dire depuis quelques semaines ou mois. L'intrigue met en cohérence l'histoire, agence les événements et les met en rapport les uns avec les autres. Elle constitue l'ordre expressif par lequel le vécu et les événements sont réunis pour former une histoire (Good, 1998, p. 299). Peter Brooks expose en ces termes sa conception de l'intrigue : «L'intrigue telle que je la conçois est le but et l'idée conductrice du récit, ce qui modèle une histoire, lui donne une direction, une volonté de signification $»^{6}$ (Brooks, 1984, p. XI). Les narrations, qu'elles appartiennent à la littérature ou au domaine historique, ont la caractéristique d'aller vers une conclusion (Ricœur, 1979; White, 1987, p. 21, p. 52). Byron Good relève que les histoires de vie des malades chroniques en Turquie, bien qu'elles soient inévitablement inachevées et incomplètes, vont vers une résolution, à savoir la guérison convoitée : "Une épreuve, donc, une volonté humaine et l'évolution des événements dans le temps vers une conclusion sont essentielles à la syntaxe des histoires » (Good, 1998, p. 301). De même, les récits rédigés pour l'OFPRA vont toujours vers la même conclusion : l'enchaînement des persécutions contraignent le requérant à quitter le Sri Lanka et à demander l'asile à un pays étranger. Toutefois, ces récits 
portent sur le passé : on expose le vécu au Sri Lanka pour convaincre les examinateurs que retourner dans leur pays mettrait leur vie en danger. De plus, ces récits sont, dans la plupart de cas, élaborés avec l'aide d'écrivains publics qui apprennent aux requérants à raconter leur histoire selon les critères de la Convention de Genève (1951). Le témoignage de Selva ne fait pas exception : dans la première partie, lui aussi, qui avait déjà travaillé son récit avec un écrivain public, narre son histoire comme s'il l'avait apprise par cœur et devait la raconter à l'OFPRA : son récit est dominé par l'angoisse de pouvoir prouver ce qu'il déclare. Ce type de récits est caractérisé par un ordre chronologique organisé autour des événements violents qu'on souhaite mettre en évidence (Mantovan, 2012) et décrit avec des détails bien précis visant à établir une crédibilité.

Dès qu'on arrive à relater le présent, l'histoire ne va plus vers une conclusion, elle reste « ouverte »; le narrateur est « au milieu du chemin » dans son parcours d'exilé, mais il ne parvient pas à " voir » son avenir : le futur est sombre, tel un point d'interrogation. Le récit se désintègre parmi ses doutes, ses peurs, sa solitude. L'intrigue disparait, il n'y a plus d'événements, on n'entrevoit plus la possibilité même d'une conclusion. L'attente de la convocation de l'OFPRA et le passé qui le tourmente sans cesse ne permettent pas à Selva d'imaginer son futur; c'est le passé qui domine: ce sont ces événements-là qui me reviennent [à l'esprit], je n'arrive pas à oublier, dit-il.

Ceux qui sont arrivés en France depuis peu n'ont pas encore eu le temps de donner du sens à leur histoire, du moins un sens autre qu'administratif, ni les ressources pour tenter de reconstruire leur monde. Ils traversent une période où tous les efforts sont dirigés vers la demande d'asile. L'incertitude à propos du résultat de leurs démarches et la souffrance causée par les traumas de la guerre, la séparation d'avec la famille et l'installation dans un nouveau pays rendent difficile de relater sa vie quotidienne avec une intrigue qui va vers une conclusion. Cette tendance s'observe davantage chez les personnes mariées que chez les jeunes célibataires, qui se projettent davantage vers l'avenir que les chefs de famille désireux de revoir le conjoint et les enfants restés dans le pays d'origine. Ce trait, enfin, concerne presque exclusivement les personnes arrivées récemment. Les autres, en France depuis plusieurs années, ont le plus souvent réélaboré leur histoire, même si elles n'ont pas de papiers.

\section{Conclusion}

La narration est un système de significations que nous utilisons dans notre négociation avec la réalité, et l'intrigue est l'élément qui met de l'ordre dans ces significations (Brooks, 1984, p. XI). La négociation que les requérants entreprennent pour la demande d'asile, décrite ici à travers l'espace de parole du COMEDE et le témoignage devant l'anthropologue, acquiert tout son sens si on élargit le regard aux structures qui gèrent la liminalité des requérants: OFPRA, CNDA, écrivains publics, centres médicaux, avocats, monde associatif contribuent tous, à leur manière, à élaborer le «visage public » des exilés d'une manière intelligible pour la société française.

Les réfugiés troublent le système international des États-nations parce qu'ils ne peuvent pas être classés selon des catégories de cet ordre. Comme l'a souligné Liisa Malkki (1995), ils sont ce que Victor Turner, se référant au moment liminaire des rites de passage, conceptualise comme un état d'" invisibilité structurelle ", car, à la fois, ils ne sont plus classifiés et pas encore classifiés (1967 : 96-7). Aux yeux des États-nations, 
les réfugiés sont perçus comme "polluants", dans le sens défini par Mary Douglas (1966) : ils représentent une catégorie associée à la marge et au désordre, constituant une menace de laquelle la société doit se protéger. Avec la mise en œuvre des politiques migratoires et du droit international, les centres de rétention administrative (CRA) et le travail de l'OFPRA et la CNDA, l'État français gère les personnes qui entrent sur son sol et demandent le droit de s'y installer durablement.

Dans la production des certificats analysée plus haut, le COMEDE, réécrivant les témoignages dans un langage médical, psychiatrique et des droits de l'homme, contribue à rendre intelligible l'histoire des requérants. De même, les récits destinés à la demande d'asile en France suivent la même forme narrative que les récits portant sur la violation des droits de l'homme, relatés dans d'autres contextes : ces narrations, en effet, commencent toujours par des violences et par la négation des droits perpétrés par des agents persécuteurs et se terminent par une réhabilitation grâce au pouvoir de la loi et de la médecine. Selon Allen Feldman, les récits post-traumatiques sont euxmêmes écrits dans une histoire, une histoire fille des Lumières et devenue de plus en plus transnationale. Ces récits sont, en effet, insérés dans de puissantes narrations (master narratives) telles que l'idée du progrès, la conciliation collective, l'évolution des droits de l'homme et de l'égalité (Feldman, 2004, pp.164-165). Ces narrations constituent le modèle, l'idéologie et le contexte auquel se conforment les mémoires des victimes pour s'organiser en histoires de vie.

À travers l'intervention des écrivains publics, du COMEDE, des institutions d'accueil et des avocats, le requérant comprend quels événements de son vécu et quels signes présents sur son corps on lui demande d'exposer; il cherche à apprendre, ainsi, à relater son histoire de la seule manière à pouvoir être reconnu comme une personne méritant de recevoir l'asile.

\section{BIBLIOGRAPHIE}

Artières, Philippe ; Farge, Arlette ; Laborie, Pierre (2002) Témoignage et récit historique, Sociétés \& Représentations, vol. 13, $\mathrm{n}^{\circ} 1$ [Disponible sur Internet]

Brooks, Peter (1984) Reading for the Plot : Design and Intention in Narrative, Oxford, Clarendon Press, $392 \mathrm{p}$.

Good, Byron (1998 [1994]) Comment faire de l'anthropologie médicale? Médecine, rationalité et vécu, Paris, Le Plessis-Robinson, $434 \mathrm{p}$.

COMEDE (2008) Guide Pratique 2008. Prise en charge médico-psycho-sociale des migrants/étrangers en situation précaire, Le Kremlin-Bicêtre, COMEDE, 569 p.

COMEDE (2011) La santé des exilés. Rapport d'activité et d'observation 2010, 71 p. [réf du 15 janvier 2017] [Disponible sur internet]

Detue, Frédérik ; Charlotte, Lacoste (2016) Ce que le témoignage fait à la littérature, Europe. Revue littéraire mensuelle, $\mathrm{n}^{\circ}$ 1041-42, pp. 3-15. 
Douglas, Mary (1966) Purity and Danger : An Analysis of Concepts of Pollution and Taboo. London, Penguin Books, 219 p.

d'Halluin-Mabillot, Estelle (2012) Les épreuves de l'asile. Associations et réfugiés face aux politiques du soupçon, Paris, Editions EHESS, 301 p.

Fassin, Didier ; d'Halluin, Estelle (2005) The Truth from the Body : Medical Certificates as Ultimate Evidence for Asylum Seekers, American Anthropologist, vol. 107, nº 4, pp. 597-608.

Fassin, Didier ; d'Halluin, Estelle (2007) Critical Evidence : The Politics of Trauma in Frech Asylum Policies, Ethos, vol. 35, n 3, pp. 300-329.

Fassin, Didier ; Rechtman, Richard (2007) L'empire du traumatisme. Enquête sur la condition de victime, Paris, Flammarion, $452 \mathrm{p}$.

Feldman, Allen (2004) Memory Theaters, Virtual Witnessing, and the Trauma-Aesthetic, Biography, n² 27, pp. 163-202.

Malkki, Liisa (1995) Purity and Exile: Violence, Memory, and National Cosmology among Hutu Refugees in Tanzania, Chicago ; London, University of Chicago Press, 374 p.

Mantovan, Giacomo (2012) Quand l'événement violent génère des récits : biographies institutionnelles de Tamouls sri lankais en France, Mondes Contemporains. Revue d'anthropologie sociale et culturelle, $\mathrm{n}^{\circ} 2$, p. 81-104.

Noiriel, Gérard (1991) La tyrannie du national. Le droit d'asile en Europe, 1793-1993, Paris, CalmannLévy, 355 p.

ONU (2011) Report of the Secretary-General's Panel of Experts on Accountability in Sri Lanka, New-York, United Nation, 214 p. [Disponible sur Internet]

Quesada, James ; Kain Hart, Laurie ; Bourgois, Philippe (2011) Structural Vulnerability and Health : Latinos Migrants Laborers in the United States, Medical Anthropology : Cross-Cultural Studies in Health and Illness, vol. 30, $\mathrm{n}^{\circ}$ 4, pp. 339-362.

Ricœur, Paul (1979) La Fonction narrative, Études Théologiques et religieuses, n54, pp. 209-230.

Turner, Victor (1991 [1967]) The Forest of Symbols : Aspects of Ndembu Ritual, Ithaca, Cornell University Press, $471 \mathrm{p}$.

White, Hayden (1987) The Content of the Form : Narrative Discourse and Historical Representation, Baltimore, Johns Hopkins University Press, 264 p.

\section{NOTES}

1. «Au milieu du chemin de notre vie, ayant quitté le chemin droit, je me trouvai dans une forêt obscure. Ah ! qu'il serait dur de dire combien cette forêt était sauvage, épaisse et âpre, la pensée seule en renouvelle la peur! »(trad. Abbaye Saint-Benoît de Port-Valais).

2. La CRR est devenue la CNDA en 2007.

3. Entretien avec des officiers de l'OFPRA et un magistrat de la CNDA, plusieurs dates.

4. L'analyse des données suivantes a été élaborée en tenant compte des travaux précédents abordant la question de l'expertise médicale (d'Halluin-Mabillot, 2012 ; Fassin et d'Halluin, 2005 ; Fassin et Rechtman, 2007).

5. Compte tenu du contexte, on pourrait penser qu'il s'agissait des LTTE. Cependant, il n'y pas de vérité officielle. Selva souligne qu'habituellement les LTTE revendiquaient les exécutions de «traîtres ", mais ici ce ne fut pas le cas. 
6. Traduction de l'anglais par Sylvette Gleize. Peter Brooks est professeur émérite de littérature comparée à Yale University.

\section{INDEX}

Index géographique : France

Mots-clés : demandeurs d'asile, accueil, accès aux soins, documents administratifs, histoire de vie, étude de cas, Tamouls

\section{AUTEUR}

\section{GIACOMO MANTOVAN}

Visiting research fellow, IASH (Edimbourg), chercheur associé (“jeune docteur"), Centre d'étude de l'Inde et de l'Asie du Sud (CEIAS) - EHESS

gmantovan@gmail.com 